\title{
GEOMETRIC INVARIANTS FOR SPARSE UNKNOWN VIEW TOMOGRAPHY
}

\author{
Mona Zehni, Shuai Huang, Ivan Dokmanić, Zhizhen Zhao \\ Department of ECE and CSL, University of Illinois at Urbana-Champaign
}

\begin{abstract}
In this paper, we study a 2D tomography problem for point source models with random unknown view angles. Rather than recovering the projection angles, we reconstruct the model through a set of rotation-invariant features that are estimated from the projection data. For a point source model, we show that these features reveal geometric information about the model such as the radial and pairwise distances. This establishes a connection between unknown view tomography and unassigned distance geometry problem (uDGP). We propose new methods to extract the distances and approximate the pairwise distance distribution of the underlying points. We then use the recovered distribution to estimate the locations of the points through constrained non-convex optimization. Our simulation results verify the robustness of our point source reconstruction pipeline to noise and error in the estimation of the features.
\end{abstract}

Index Terms - Point source model, rotation-invariant features, 2D tomography, unassigned distance geometry.

\section{INTRODUCTION}

We consider the following forward model,

$$
\begin{aligned}
s_{\ell}[u] & =\mathcal{D}\left\{\mathcal{P}_{\theta_{\ell}} I\right\}[u]+\varepsilon_{\ell}[u], \quad \ell \in\{1,2, \ldots, L\} \\
I(x, y) & =\sum_{k=1}^{K} \delta\left(x-x_{k}, y-y_{k}\right)
\end{aligned}
$$

where $I$ is an unknown model consisting of point sources (i.e. Dirac delta distributions) located at $\left\{\left(x_{k}, y_{k}\right)\right\}_{k=1}^{K} \in \mathbb{R}^{2}$, and $\mathcal{D}$ is the discretization operator. For the sake of simplicity, we assume unit weights for all the point sources. The operator $\mathcal{P}_{\theta}$ is the $1 \mathrm{D}$ projection along direction $\theta$, where $\theta$ is the angle between the projected direction and the horizontal $x$ axis. We assume that $\theta_{\ell}$ is a sample drawn from a uniform distribution over $[0,2 \pi)$ which is a realistic assumption in many scenarios. We introduce the sampling operator $\mathcal{D}$ to take into account of the finite resolution of the digitized projection data,

$$
\mathcal{D}(f)[u]=\int_{\left(u-\frac{1}{2}\right) \Delta}^{\left(u+\frac{1}{2}\right) \Delta} f(x) d x, \text { for } u \in\{-M, \ldots, M\},
$$

where $\Delta$ is the sampling step-size. The observed signals are contaminated by additive white Gaussian noise, i.e. $\varepsilon_{\ell}[u] \sim \mathcal{N}\left(0, \sigma^{2}\right)$. Fig. 1 illustrates the observation model.

Our goal is to recover the point source model from $\left\{s_{\ell}\right\}_{\ell=1}^{L}$. The problem of recovering a point-source signal arises in array signal processing [1], compressed sensing [2], super-resolution [3], radio astronomy [4, 5], unassigned distance geometry [6, 7], imaging

This work is partially supported by National Science Foundation under Grant CIF-1817577. molecules using X-ray crystallography [8], powder diffraction [9] and cryo-electron microscopy (EM) atomic modeling [10], to name a few.

The state of the art approaches in 2D tomography from known projection angles mainly recover the underlying image by solving a regularized least squares problem [11, 12]. The regularization then imposes the structure of the image, for examples its smoothness or sparsity. The main assumption of these methods is the availability of the projection angles. On the other hand, 2D tomography from unknown projection angles is addressed in $[13,14,15]$ which aim at recovering the projection angles from the projection data and then invert the tomography operation to recover the image. However, these methods do not incorporate the model of the underlying image, for example, sparsity in our parametric image model.

In this paper we propose a pipeline that recovers a $2 \mathrm{D}$ point source model directly from a set of projection lines taken at random unknown angles. Our method consists of three steps, 1) constructing rotation-invariant features from the projection data, 2) estimating distances and distance distributions from the rotation-invariant features, and 3) reconstruction of the point source model from the estimated distance distributions. Through the use of rotation-invariant features, we circumvent the recovery of the projection angles. We show that the estimated features are expressed as a summation of zeroth-order Bessel functions of the first kind. We then use the properties of Bessel functions to estimate the unlabeled radial/pairwise distances values and their distance distributions, which can be efficiently computed using the proposed methods. Specifically, the unlabeled radial distance distribution describes the distribution of the distances from the point sources to the origin, i.e. the center of mass. The unlabeled pairwise distance distribution, also known as pair distribution function (PDF), describes the probability distribution of the pairwise distances between any two point sources. In the final step of our pipeline, following the same line as [16], we recover the point source model from the estimated distance distributions by solving a constrained nonconvex optimization problem. The proposed pipeline is robust to noise if the number of samples are sufficiently large.

\section{METHOD}

We start by elaborating upon the generation of the invariant features from the projection data. Next, we describe two methods that target estimating the distances and the distribution of the distances. Finally, we describe the point source model reconstruction via the estimated distance distributions.

\subsection{Estimating the rotational invariant features}

We estimate a set of rotational invariant features from the observations $\left\{s_{\ell}\right\}_{\ell=1}^{L}$. These features are functions of certain geometric information of the point source model, namely, the radial distances 

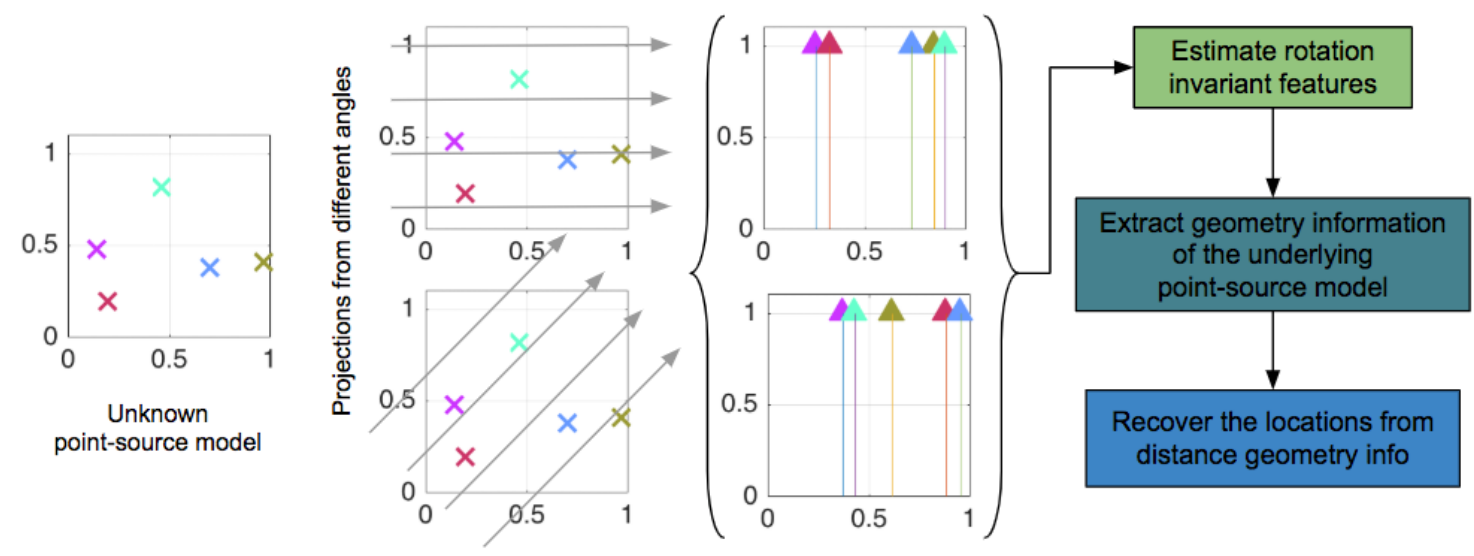

Collection of 1D projections

taken from unknown angles

Fig. 1. The 2D point source localization pipeline. Crosses indicate point sources; triangles indicate Dirac deltas in 1D.

from the points to the origin and the pairwise distances between any two points. We assume that the center of mass is at the origin and all pairwise distances are smaller than $R$. All points lie within a disk of radius $R$. Since the point source model $I$ is the summation of Dirac deltas, its projections from different angles also consist of Dirac deltas,

$$
\left(\mathcal{P}_{\theta} I\right)(r)=\sum_{k=1}^{K} \delta\left(r-\left(y_{k} \cos \theta-x_{k} \sin \theta\right)\right) .
$$

The observation is digitized into $2 M+1$ bins and the discretization operator $\mathcal{D}$ applied to (3) leads to,

$$
g_{\theta}[u]=\left(\mathcal{D} \mathcal{P}_{\theta} I\right)[u]=\sum_{k=1}^{K} \frac{\mathbb{1}_{y_{k} \cos \theta-x_{k} \sin \theta}}{\Delta} \in\left[u-\frac{1}{2}, u+\frac{1}{2}\right],
$$

where $u \in\{-M, \ldots, M\}$ and $\Delta=\frac{2 R}{2 M+1}$. In order to derive the rotation-invariant features from (4), we first take the discrete Fourier transform (DFT) of a projection line at angle $\theta$,

$$
\hat{g}_{\theta}[\nu]=\sum_{k=1}^{K} \exp \left(\imath \frac{2 \pi \nu}{(2 M+1)}\left\lfloor\frac{y_{k} \cos \theta-x_{k} \sin \theta}{\Delta}\right\rceil\right),
$$

where $\lfloor\cdot 7$ denotes the nearest integer. For sufficiently small $\Delta$, we can approximate (5) without the rounding. Assuming the view angles are uniformly distributed, the rotation-invariant features are defined as:

$$
\mu[\nu]=\mathbb{E}_{\theta}\left\{\hat{g}_{\theta}[\nu]\right\} \approx \sum_{k=1}^{K} J_{0}\left(\frac{\pi r_{k}}{R} \nu\right),
$$

where $J_{0}(\cdot)$ is the zeroth order Bessel function of the first kind. The radial distance of the $k$-th point source is denoted by $r_{k}$, i.e., $r_{k}=\sqrt{x_{k}^{2}+y_{k}^{2}}$. Following the same procedure, we derive the power spectral density as,

$$
\begin{aligned}
\mathbb{E}_{\theta}\left\{\left|\hat{g}_{\theta}[\nu]\right|^{2}\right\} & \approx \sum_{m=1}^{K} \sum_{n=1}^{K} J_{0}\left(\frac{\pi d_{m, n}}{R} \nu\right) \\
& =K J_{0}(0)+2 \sum_{m=1}^{K} \sum_{n=m+1}^{K} J_{0}\left(\frac{\pi d_{m, n}}{R} \nu\right)
\end{aligned}
$$

where $d_{m, n}$ represents the distance between the $m$-th and $n$-th point source, $d_{m, n}=d_{n, m}=\sqrt{\left(x_{n}-x_{m}\right)^{2}+\left(y_{n}-y_{m}\right)^{2}}$.
Therefore, we define the second-order invariant feature as,

$$
C[\nu]=\left(\mathbb{E}_{\theta}\left\{\left|\hat{g}_{\theta}[\nu]\right|^{2}\right\}-K\right) / 2 .
$$

We estimate the features in (6) and (8) from the observations $\left\{s_{\ell}[u]\right\}_{\ell=1}^{L}$ as,

$$
\begin{aligned}
& \widehat{\mu}[\nu]=\frac{1}{L} \sum_{\ell=1}^{L} \hat{s}_{\ell}[\nu], \\
& \widehat{C}[\nu]=\left(\frac{1}{L} \sum_{\ell=1}^{L}\left|\hat{s}_{\ell}[\nu]\right|^{2}-(2 M+1) \sigma^{2}-K\right) / 2,
\end{aligned}
$$

where $\hat{s}_{\ell}[\nu]$ is an empirical realization of $\hat{g}_{\theta}[\nu]$, and the subtraction of $(2 M+1) \sigma^{2}$ in (10) serves to debias the estimation of $C$. By the law of large numbers, the sample estimates $\hat{\mu}$ and $\hat{C}$ converge to $\mu$ and $C$ when the sample size $L \rightarrow \infty$. The features are invariant to the global rotation of the point sources.

\subsection{Recovering the geometry information of the model}

We propose two methods to extract the geometric information of the model from the invariant features.

- Prony-based distance estimation (PBDE): In our first method, we make use of the asymptotic behavior of the Bessel function [17, p.364],

$$
J_{0}(z) \approx \sqrt{\frac{2}{\pi z}} \cos \left(z-\frac{\pi}{4}\right)
$$

for $z \gg 1 / 4$, to extract $\left\{r_{k}\right\}_{k=1}^{K}$ and $\left\{d_{m, n}\right\}_{m, n=1}^{K}$ using Prony's method. Based on (11), for sufficiently large integer $\nu$, the features can be approximated by,

$$
\widehat{\mu}[\nu] \approx \sum_{k=1}^{K} \frac{e^{\imath\left(a_{k} \nu-\pi / 4\right)}+e^{-\imath\left(a_{k} \nu-\pi / 4\right)}}{\sqrt{2 \pi a_{k} \nu}}
$$

where $a_{k}=\frac{\pi r_{k}}{R}$, for integer $\nu \gg\left\lceil\frac{1}{4 a_{k}}\right\rceil$. We scale $\widehat{\mu}[\nu]$ by $\sqrt{\nu}$ and the new features $\sqrt{\nu} \widehat{\mu}[\nu]$ are approximated by the sum of $2 K$ complex exponentials. Thus, we apply Prony's method to determine the filter that annihilates $\widehat{\mu}$. Arguments of the complex zeros of this filter correspond to the radial distances. We can use the same procedure to recover the pairwise distances from $\widehat{C}$. 
- Distance distribution estimator (DDE): In our second method, rather than recovering the distances explicitly, we derive a distance distribution function that is later used to recover the locations of the point sources. For this purpose, we rely on the orthogonality properties of the Bessel functions,

$$
\delta(u-v)=u \int_{0}^{\infty} t J_{0}(u t) J_{0}(v t) d t, \forall v \in \mathbb{R} .
$$

In order to implement (13), we replace the infinite upper limit of the integral with a frequency cutoff $c$,

$$
\int_{0}^{c} t J_{0}(u t) J_{0}(v t) d t=c \frac{u J_{1}(u c) J_{0}(v c)-v J_{0}(u c) J_{1}(v c)}{u^{2}-v^{2}} .
$$

The integral (14) is evaluated using the Gauss-Legendre quadrature rule [18, Chap. 4] and we perform Hankel transform on the features $\mu$ and $C$,

$$
\begin{gathered}
f_{\mu}\left(u_{j}\right)=\int_{0}^{c} \mu(t) J_{0}\left(u_{j} t\right) t d t \approx \sum_{i=1}^{n} w\left(t_{i}\right) t_{i} \widehat{\mu}\left(t_{i}\right) J_{0}\left(u_{j} t_{i}\right), \\
f_{C}\left(u_{j}\right)=\int_{0}^{c} C(t) J_{0}\left(u_{j} t\right) t d t \approx \sum_{i=1}^{n} w\left(t_{i}\right) t_{i} \widehat{C}\left(t_{i}\right) J_{0}\left(u_{j} t_{i}\right),
\end{gathered}
$$

with $n$ points $\left\{t_{i}\right\}_{i=1}^{n}$ on the interval $[0, c]$ and the associated weights $w\left(t_{i}\right)$. The Fourier coefficients at the non-equally spaced quadrature points are evaluated from the projection lines using the non-uniform FFT [19]. We define the approximated distributions of the radial distances and the pairwise distances in (16) as,

$$
p_{\mu}\left(u_{j}\right)=\frac{\left|f_{\mu}\left(u_{j}\right)\right|^{2}}{\sum_{j=1}^{l}\left|f_{\mu}\left(u_{j}\right)\right|^{2}}, p_{C}\left(u_{j}\right)=\frac{\left|f_{C}\left(u_{j}\right)\right|^{2}}{\sum_{j=1}^{l}\left|f_{C}\left(u_{j}\right)\right|^{2}},
$$

where $l$ is the total number of equally spaced points on the real line, and use $p_{\mu}$ and $p_{C}$ to recover the point source model in the next subsection.

\subsection{Point source recovery from distance distribution}

After the radial and pairwise distance distributions are computed, we can recover the locations of the point sources using the approach proposed by [16]. The 2D domain space $\mathbb{R}^{2}$ is first divided into $m$ unit cells $\left\{c_{i}\right\}_{i=1}^{m}$. The point source locations are then represented by an indicator vector $\boldsymbol{z} \in\{0,1\}^{m}$ where each cell corresponds to a possible point source location. Each entry $z_{i}$ of $\boldsymbol{z}$ corresponds to a unit cell $c_{i}$ in the domain space $\mathbb{R}^{2} ; z_{i}=0$ implies that no point source occupies the $c_{i}$ cell, while $z_{i}=1$ marks the existence of a point in the corresponding cell.

Based on this definition, the pairwise distance distribution $Q(d)$ can be written in closed-form with respect to $z$ :

$$
Q(d)=\frac{1}{m^{2}} \boldsymbol{z}^{\mathrm{T}} \boldsymbol{A}_{d} \boldsymbol{z}
$$

where $\boldsymbol{A}_{d} \in\{0,1\}^{m \times m}$ is a symmetric Toeplitz matrix. Let $d_{c_{i}, c_{j}}$ denote the distance between the $i$-th cell $c_{i}$ and the $j$-th cell $c_{j}$. The $(i, j)$-th entry of $\boldsymbol{A}_{d}$ is determined by

$$
A_{d}(i, j)= \begin{cases}1, & \text { if } d_{c_{i}, c_{j}}=d \\ 0, & \text { if } d_{c_{i}, c_{j}} \neq d\end{cases}
$$

Algorithm 1 Point source recovery from distance distribution

Input: Projection data, $\left\{s_{\ell}[u]\right\}_{\ell=1}^{L}$

Output: The estimated $\left\{x_{k}, y_{k}\right\}_{k=1}^{K}$

1: Estimate the distance distributions, $p_{\mu}$ and $p_{C}$, from $\left\{s_{\ell}[u]\right\}_{\ell=1}^{L}$ using (10) and DDE.

2: Incorporate $p_{\mu}$ and $p_{C}$ to formulate (19) and solve it using projected gradient descent.

Table 1. Success rate (\%) of PBDE in recovering the radial distances

\begin{tabular}{c|cccc}
\multicolumn{5}{c}{$K=5$} \\
\multicolumn{1}{c|}{} & & & & \\
SNR & $\infty$ & 100 & 10 & 1 \\
\hline 100 & 95 & 88 & 80 & 53 \\
500 & 99 & 90 & 76 & 50 \\
1000 & 98 & 90 & 80 & 56 \\
1500 & 98 & 89 & 77 & 52
\end{tabular}

\begin{tabular}{c|cccc}
\multicolumn{5}{c}{$K=10$} \\
${ } }$ & $\infty$ & 100 & 10 & 1 \\
\hline 100 & 49 & 47 & 43 & 36 \\
500 & 65 & 53 & 48 & 38 \\
1000 & 72 & 55 & 49 & 38 \\
1500 & 76 & 52 & 48 & 38
\end{tabular}

The pairwise distance distribution $p_{C}(d)$ is extracted from the projection lines using (16). Now the target is to find an indicator $\boldsymbol{z}$ such that its corresponding pairwise distance distribution $Q(d)$ matches $p_{C}(d)$. For this purpose, as proposed in [16], we relax the integer constraint on $\boldsymbol{z}$ and minimize the nonconvex cross entropy between $p_{C}(d)$ and $Q(d)$ subject to a set of convex constraints,

$$
\begin{aligned}
\min _{\boldsymbol{z}} & -\sum_{d} p_{C}(d) \log Q(d) \\
\text { subject to } & \|\boldsymbol{z}\|_{1}=n \text { and } z_{i} \in[0,1], \forall i \\
& \boldsymbol{R} \boldsymbol{z}=\boldsymbol{r} \\
& \boldsymbol{P} \boldsymbol{z}=\boldsymbol{s},
\end{aligned}
$$

where $\boldsymbol{R}$ is a matrix such that $\boldsymbol{R} \boldsymbol{z}=\boldsymbol{r}$ enforces the radial distance distribution $p_{\mu}(d)$, represented by the probability vector $\boldsymbol{r}$; while $\boldsymbol{P}$ denotes the projection operator that relates the point source model $\boldsymbol{z}$ to one of the observed projection lines $\boldsymbol{s}$. The matrix $\boldsymbol{R}$ is derived similarly as (18). We use projected gradient descent to solve (19).

\section{NUMERICAL RESULTS}

To test the algorithm performance, we generate the coordinates of $K$ points randomly on $[-1,1] \times[-1,1]$. To generate the features, we take uniformly distributed $L=10^{4}$ projections of the point source model. We generate 100 random point source realizations. The invariant features are computed from (10), followed by (15) and (16) in order to get the transformed features and an estimation of the distance distribution. Also, we define signal to noise ratio (SNR) of

Table 2. Success rate (\%) of the point source recovery from distance distribution

\begin{tabular}{c|ccccc|ccccc}
\multicolumn{9}{c}{$K=5$} \\
\multicolumn{1}{c|c}{} & $\infty$ & 100 & 10 & 1 & 0.5 & $\infty$ & 100 & 10 & 1 & 0.5 \\
\hline 100 & 25 & 28 & 26 & 22 & 7 & 0 & 0 & 0 & 0 & 0 \\
500 & 95 & 94 & 93 & 93 & 81 & 75 & 73 & 73 & 73 & 47 \\
1000 & 97 & 96 & 96 & 94 & 80 & 80 & 81 & 82 & 75 & 49 \\
1500 & 95 & 97 & 95 & 98 & 90 & 92 & 92 & 91 & 90 & 58
\end{tabular}



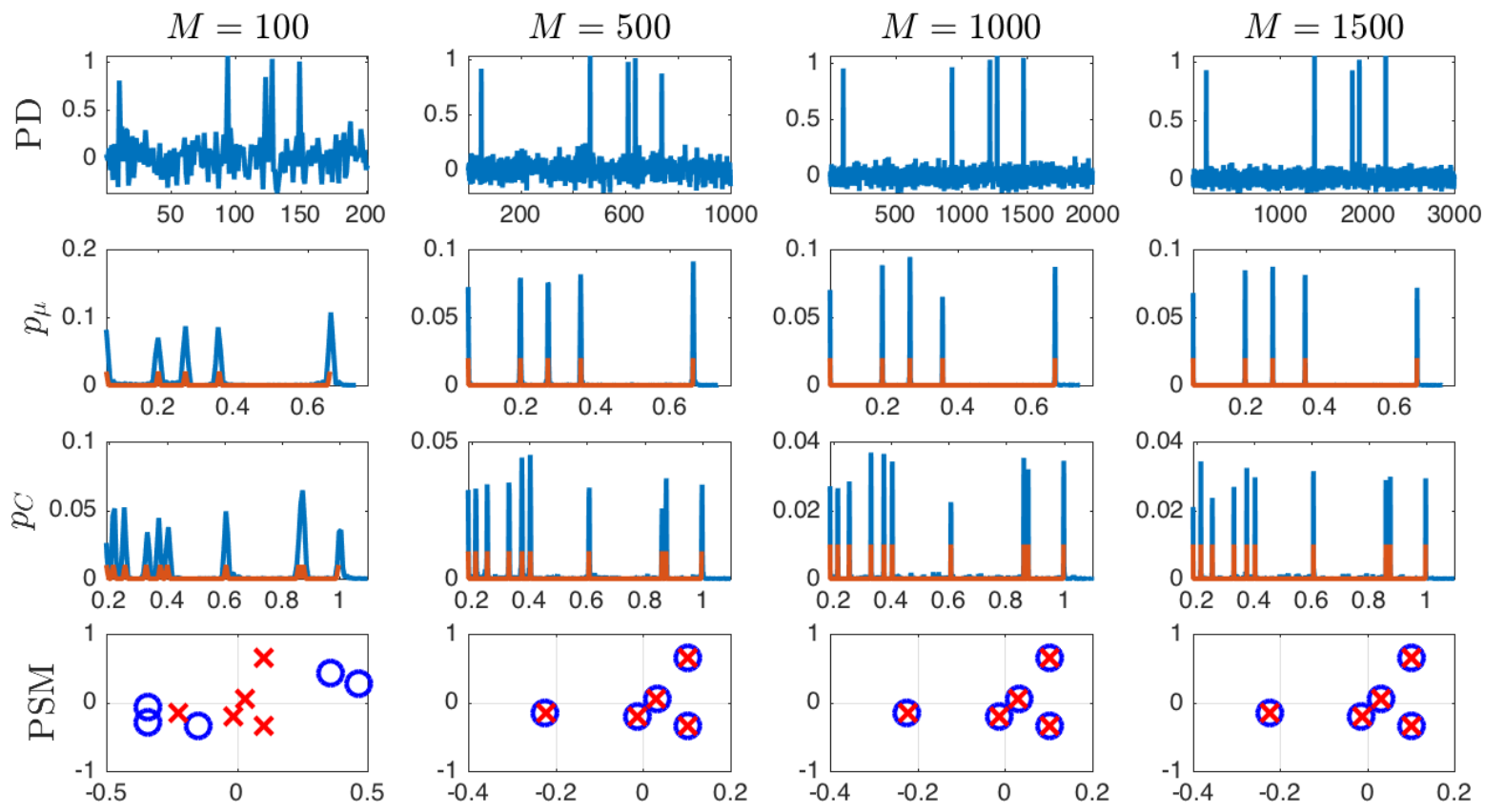

Fig. 2. An illustration of the projection data (PD, the first row) for $\mathrm{SNR}=1$, the estimated distribution of the radial and pairwise distances (16) (the second and third row), a comparison of the true and the recovered point source models (PSM, the last row). In the second and third row, the blue and orange curves represent the true and estimated distance distribution (up to a scaling factor). In the last row, the blue circles and red crosses mark the recovered and true point source models.

the projection data as the average power of the clean projection line divided by $\sigma^{2}$.

We use earth mover's distance (EMD) [20] to quantify the performance of our methods. EMD is a measure of the distance between two probability distributions. We say a recovery is successful if the EMD between the recovered distance distribution and the true one is smaller than a threshold $t h=0.1$. Accordingly, we define success rate as the portion of trials for which successful recovery is achieved,

$$
\text { success-rate }=P\{\operatorname{EMD}(p, q) \leq t h\}
$$

where $p$ and $q$ mark the true and the recovered distributions.

\subsection{Discussion of the results}

There are two ways to extract geometric features from the random projections:

- Extracting the radial distances using PBDE: Table 1 present the success rate in the recovery of radial distances for $K=5$ and $K=10$ respectively for different values of SNR and $M$. In order for (11) to be a good approximation, we choose $\nu \geq 10$. For the Prony based method, $M$ denotes the number of discretizations of the projection lines. Thus, larger $M$ means finer discretization of the projection lines. The results suggest that as $K$ increases, it is harder to extract the radial distances due to denser distance distributions. On the other hand, as long as the approximation in (11) is accurate, changing $M$ does not significantly affect the performance. Furthermore, the higher the SNR, the higher the success rate. This happens as a result of more accurate estimation of the features from the projection data.

- The performance of the point source reconstruction: For this experiment, the estimated features $\widehat{\mu}$ and $\widehat{C}$ are transformed by (15). Then we use $p_{\mu}$ and $p_{C}$ defined in (16) and solve the optimization problem (19) using projected gradient descent to find the locations of the points. Fig. 2 summarizes the observations, approximate distance distribution and the final results of our pipeline for a setting of $K=5$ and SNR $=1$. Note that although the projection data (first row) are severely contaminated by noise, the features (second and third row) are estimated accurately and match features from the true point source model very well (up to a scaling factor). In addition, coarser discretization of the projection data (smaller $M$ ) reduces the accuracy in extracting the geometric invariants and localizing the points (see the first column of Fig. 2).

Table 2 shows the success rates in the point source recovery of the model from distance distributions. The results suggest that 1) it is critical to have a large $M$ in order to have a good approximation of the transformed features in (16),2) our pipeline is robust to various noise levels on the projection data, 3) with larger $K$ the success rate decreases, because the minimum separation between points become smaller.

\section{CONCLUSION}

In this paper, we proposed a pipeline to recover a point source model from a set of projections taken from unknown angles. Instead of first recovering the angles and then solving a tomography problem, we directly recover the locations of the point sources using a set of rotational invariant features that are estimated from the projection data. The features contain geometric information about the point source model: the unlabeled radial/pairwise distances values extracted by the Prony-based distance estimation method, and their distance distributions recovered by the distance distribution estimator method. Finally, based on the recovered distance distributions, the locations of the point sources are reconstructed. We show that the proposed approach is robust to additive white Gaussian noise for various simulation set-ups. 


\section{REFERENCES}

[1] H. Krim and M. Viberg, "Two decades of array signal processing research: the parametric approach," IEEE Signal Processing Magazine, vol. 13, no. 4, pp. 67-94, July 1996.

[2] H. Boche, R. Calderbank, G. Kutyniok, and J. Vybíral, A Survey of Compressed Sensing, pp. 1-39, Springer International Publishing, Cham, 2015.

[3] E. J. Candès and C. Fernandez-Granda, "Towards a mathematical theory of super-resolution," Communications on Pure and Applied Mathematics, vol. 67, no. 6, pp. 906-956, 2014.

[4] H. Pan, T. Blu, and M. Vetterli, "Towards generalized FRI sampling with an application to source resolution in radioastronomy," IEEE Transactions on Signal Processing, vol. 65, no. 4, pp. 821-835, Feb 2017.

[5] H. Pan, M. Simeoni, P. Hurley, T. Blu, and M. Vetterli, "LEAP: Looking beyond pixels with continuous-space EstimAtion of Point sources," Astronomy and Astrophysics, vol. 608, pp. A136, Dec. 2017.

[6] S. J. L. Billinge, P. M. Duxbury, D. S. Gonçalves, C. Lavor, and A. Mucherino, "Assigned and unassigned distance geometry: applications to biological molecules and nanostructures," $4 O R$, vol. 14, no. 4, pp. 337-376, Dec 2016.

[7] P. Duxbury, L. Granlund, S. Gujarathi, P. Juhas, and S. Billinge, "The unassigned distance geometry problem," Discrete Appl. Math., vol. 204, no. C, pp. 117-132, May 2016.

[8] D. Jan and J. Mesters, Principles of Protein X-Ray Crystallography, Springer New York, 2007.

[9] F. Gramm, C. Baerlocher, L. B. McCusker, S. J. Warrender, P. A. Wright, B. Han, S. B. Hong, Z. Liu, T. Ohsuna, and O. Terasaki, "Complex zeolite structure solved by combining powder diffraction and electron microscopy," Nature, vol. 444, 2006.

[10] S. H. Scheres, "Relion: Implementation of a bayesian approach to cryo-em structure determination," Journal of Structural Biology, vol. 180, no. 3, pp. 519 - 530, 2012.

[11] L. Donati, M. Nilchian, S. Trpout, C. Messaoudi, S. Marco, and M. Unser, "Compressed sensing for stem tomography," Ultramicroscopy, vol. 179, pp. 47 - 56, 2017.

[12] M. Nilchian, C. Vonesch, S. Lefkimmiatis, P. Modregger, M. Stampanoni, and M. Unser, "Constrained regularized reconstruction of x-ray-dpci tomograms with weighted-norm," Opt. Express, vol. 21, no. 26, pp. 32340-32348, Dec 2013.

[13] S. Basu and Y. Bresler, "Feasibility of tomography with unknown view angles," IEEE Transactions on Image Processing, vol. 9, no. 6, pp. 1107-1122, June 2000.

[14] S. Basu and Y. Bresler, "Uniqueness of tomography with unknown view angles," IEEE Transactions on Image Processing, vol. 9, no. 6, pp. 1094-1106, June 2000.

[15] R. R. Coifman, Y. Shkolnisky, F. J. Sigworth, and A. Singer, "Graph laplacian tomography from unknown random projections," IEEE Transactions on Image Processing, vol. 17, no. 10, pp. 1891-1899, Oct 2008.

[16] S. Huang and I. Dokmanić, "Reconstructing point sets from distance distributions," CoRR, vol. abs/1804.02465, 2018.

[17] M. Abramowitz and I. A. Stegun, Bessel Functions $J$ and $Y$, pp. 358-364, Dover, 1972.
[18] W. H. Press, S. A. Teukolsky, W. T. Vetterling, and B. P. Flannery, "Numerical recipes in fortran 77, vol. 1," New York, NY: Press Syndicate of the University of Cambridge, 1992.

[19] L. Greengard and J.-Y. Lee, "Accelerating the nonuniform fast fourier transform," SIAM review, vol. 46, no. 3, pp. 443-454, 2004.

[20] Y. Rubner, C. Tomasi, and L. J. Guibas, "The earth mover's distance as a metric for image retrieval," International Journal of Computer Vision, vol. 40, no. 2, pp. 99-121, Nov 2000. 